\title{
Increased STREM-1 in pregnant women with premature rupture of membranes and subclinical chorioamnionitis
}

\author{
JIANPING WANG $^{1}$, YIJI ZHANG ${ }^{1}$, HANQING ZHANG ${ }^{2}$, WANGLEI QU ${ }^{1}$, \\ JIEQIANG LV ${ }^{1}$, YUHUAN WANG ${ }^{1}$ and JIAN XIAO ${ }^{2}$ \\ ${ }^{1}$ Department of Gynecology and Obstetrics, The Second Affiliated Hospital; ${ }^{2}$ School of Pharmacy, \\ Wenzhou Medical College, Wenzhou, Zhejiang 325035, P.R. China
}

Received May 17, 2011; Accepted August 10, 2011

DOI: $10.3892 / \mathrm{mmr} .2011 .710$

\begin{abstract}
Premature rupture of membranes (PROM) is a common obstetric complication frequently occurring with concomitant chorioamnionitis. The present study aimed to evaluate levels of soluble triggering receptor expressed on myeloid cells-1 (sTREM-1) in serum and amniotic fluid in pregnant women with PROM and to compare sTREM-1 with the commonly used laboratory indicators, serum C-reactive protein (CRP) and white blood cell (WBC) count. A total of 55 pregnant women with PROM were enrolled. Their venous blood and amniotic fluid were collected at delivery. sTREM-1 concentrations in the serum and amniotic fluid were determined by enzyme-linked immunosorbent assay. The measured data were compared with the pathological results of the placenta and fetal membrane. Meanwhile, sTREM-1 was compared with the laboratory indicators, serum CRP and WBC count. Serum and amniotic fluid sTREM-1 levels were significantly higher in pregnant women with subclinical chorioamnionitis compared to pregnant women without chorioamnionitis. Serum concentration of STREM-1 yielded a sensitivity of $81.8 \%$ and a specificity of $77.3 \%$ for the prediction of subclinical chorioamnionitis. The amniotic fluid concentration of sTREM-1 resulted in a sensitivity of $81.8 \%$ and a specificity of $86.4 \%$ for the prediction of subclinical chorioamnionitis. In conclusion, serum and amniotic fluid sTREM-1 levels may emerge as early biological indicators for predicting PROM complicated with subclinical chorioamnionitis. sTREM-1 levels are superior to WBC count in predicting subclinical chorioamnionitis.
\end{abstract}

Correspondence to: Dr Yuhuang Wang, Department of Gynecology and Obstetrics, The Second Affiliated Hospital, Wenzhou Medical College, Wenzhou, Zhejiang 325035, P.R. China E-mail: wangqu61@126.com

Dr Jian Xiao, School of Pharmacy, Wenzhou Medical College, Wenzhou, Zhejiang 325035, P.R. China

E-mail: xfxj2000@126.com

Key words: subclinical chorioamnionitis, premature rupture of membranes, soluble triggering receptor expressed on myeloid cells-1 white blood cell, C-reactive protein

\section{Introduction}

Premature rupture of membranes (PROM) refers to the rupture of membranes occurring prior to the onset of labor. As a common obstetric complication, it occurs in $2.7-17 \%$ of total deliveries and its incidence continues to rise (1). The mechanisms of PROM are not fully clear, and major causes responsible for PROM include infection, structural abnormalities of the fetal membrane, amniotic villus degeneration and micronutrient deficiencies. Although the etiology of PROM is multifactorial, epidemiological, clinical and molecular biology data have confirmed that ascending infection-caused genital tract inflammation plays a major role in the occurrence of PROM (2). Approximately $60 \%$ of PROM cases have concomitant chorioamnionitis, and infection is proportionately more common when fewer gestational weeks have elapsed at the time of PROM occurrence (3). Prolonged duration of PROM boosts the risk of infection $(4,5)$. Romero et al reported that the positive rate of bacterial culture using samples from transabdominal amniocentesis of PROM patients was $38 \%$ (6). It is now widely accepted that PROM and premature labor are a group of clinical syndromes closely associated with infection of the amniotic cavity.

PROM complicated with chorioamnionitis is a common cause of perinatal infant mortality. It may lead to intrauterine infection, preterm labor of fetuses and low survival rate. In addition, it may result in maternal puerperal infection and even sepsis. It is difficult to carry out early diagnosis of PROM complicated with subclinical chorioamnionitis. Currently, white blood cell (WBC) count and serum C-reactive protein (CRP) are commonly used to monitor intrauterine infection, but a lack of sufficient sensitivity and specificity limits this application.

In recent years, triggering receptor expressed on myeloid cells-1 (TREM-1) has drawn increasing attention as a newly discovered significant marker for diagnosis of inflammatory diseases. TREM-1 exists in two forms: surface TREM-1 and soluble TREM-1 (sTREM-1). During infection, sTREM-1 may be released into body fluid and is closely related to the severity of infection. Therefore, sTREM-1 may be used as a significant diagnostic indicator of inflammation. By determining serum and amniotic fluid levels of sTREM-1 in pregnant women with PROM, the present study aimed to examine the clinical value of levels of serum and amniotic fluid sTREM-1 for early diagnosis 
of PROM and the correlation between serum STREM-1 and amniotic fluid sTREM-1, and to compare serum and amniotic fluid sTREM-1 with WBC count and serum CRP, the two commonly employed indicators for infection monitoring.

\section{Materials and methods}

Study subjects. A population comprising 55 singleton pregnant women with PROM who underwent perinatal care and gave childbirth at the Maternity Department of The Second Affiliated Hospital of Wenzhou Medical College between February and August 2009 were enrolled. They exhibited no other obstetrical, medical or surgical complications such as high blood pressure, heart disease, diabetes, hepatitis, nephritis, pneumonia, autoimmune diseases or cancer. The diagnosis of PROM was established using the following criteria: i) pregnant women felt a sudden and relatively copious discharge of vaginal fluid and its flow increased when the presenting part of the fetus was pushed upward with fingers; ii) amniotic fluid accumulated at the posterior vaginal fornix or discharge of amniotic fluid from the orifice of the uterus was observed using vaginal speculum examination; iii) $\mathrm{pH}$ of vaginal fluid $\geq 6.5$ was considered positive; iv) frond-like crystals observed upon vaginal fluid examination were amniotic fluid (7). This study was approved by the Ethics Committee of The Second Affiliated Hospital of Wenzhou Medical College, and informed consent was obtained from all pregnant women prior to participation.

Sample collection and treatment. The vulva, vagina and cervix of pregnant women about to give birth were routinely disinfected. After the vagina was expanded using a vaginal speculum, $3 \mathrm{ml}$ of amniotic fluid were withdrawn using a needle-free syringe while the fundus was pressed by hand. The supernatant was kept following centrifugation at $3000 \mathrm{rpm}$ for $10 \mathrm{~min}$. Meanwhile, $6 \mathrm{ml}$ of cubital venous blood was withdrawn and separated into two tubes. A total amount of $3 \mathrm{ml}$ of the cubital venous blood was placed into a test tube containing no anti-coagulant and centrifuged at $3000 \mathrm{rpm}$ for $10 \mathrm{~min}$. The serum was maintained at $-80^{\circ} \mathrm{C}$ alongside the supernatant of the amniotic fluid. Subsequently, sTREM-1 levels in the serum and amniotic fluid were determined using the sTREM-1 enzyme-linked immunosorbent assay kit purchased from R\&D Systems (Minneapolis, MN, USA) in accordance with the manufacturer's instructions. The other $3 \mathrm{ml}$ of venous blood was placed into an ethylenediamine tetra-acetic acidanticoagulated tube to detect the WBC count and CRP levels. A narrow strip of fetal membrane was cut directly from the rupture of the fetal membrane until the edge of the placenta after delivery. The harvested fetal membrane was rolled up, pinned, fixed in $10 \%$ formaldehyde, and underwent $\mathrm{H} \& \mathrm{E}$ staining for pathological examination. The pathological diagnostic criterion of chorioamnionitis was the presence of $\geq 5$ infiltrated neutrophils in each high power field under microscopy of the pathological sections of fetal membranes (8).

Statistical analysis. Data were processed using the SPSS 11.5 statistical package. The Shapiro-Wilk or Kolmogorov-Smirnov tests were used to test the normality of the data. As the data were in a non-normal distribution, results are presented as

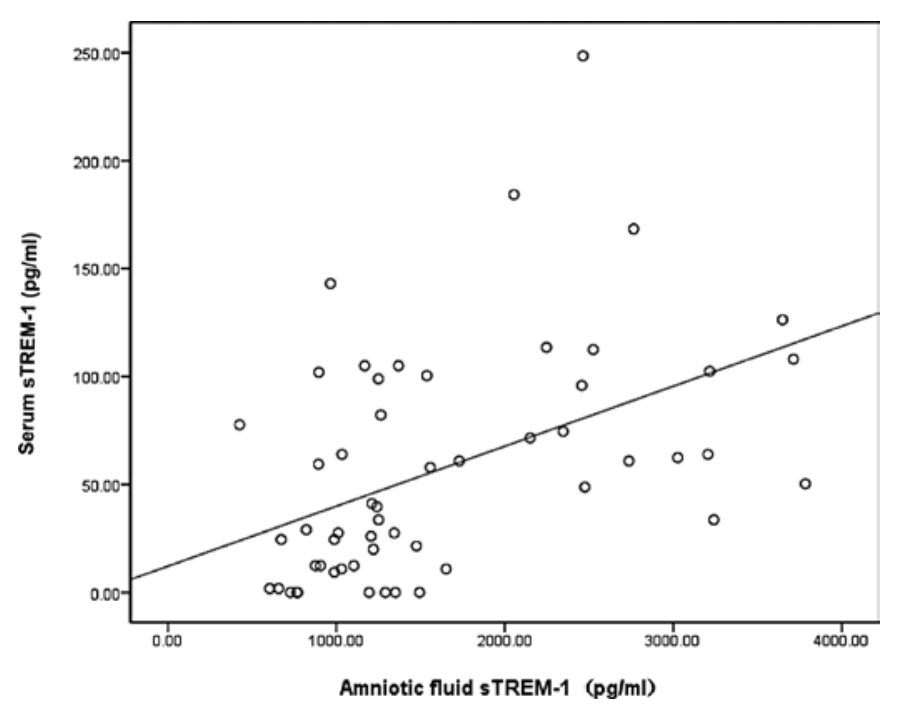

Figure 1. Correlation analysis of serum soluble triggering receptor expressed on myeloid cells-1 (sTREM-1) and amniotic fluid sTREM-1 levels $\left(\mathrm{r}_{\mathrm{s}}=0.513\right.$, $\mathrm{P}<0.01)$.

medians (interquartile range). The Mann-Whitney U test and Spearman rank correlation analysis were performed according to the type of the data. The areas under the receiver operating characteristic (ROC) curve (AUC) and cutoff values were determined using ROC curve analysis. $\mathrm{P}<0.05$ was considered statistically significant.

\section{Results}

General information. Among the 55 pregnant women with PROM (including 33 term and 22 preterm pregnant women), $33(60 \%)$ had chorioamnionitis, as revealed by histological examination of the fetal membranes. Accordingly, the 55 pregnant women with PROM were divided into chorioamnionitis and non-chorioamnionitis groups according to the pathology of the fetal membranes. There was no statistical difference in age, gestational age, or birth weight between the two groups $(\mathrm{P}>0.05)$ (Table I).

Serum and amniotic fluid concentrations of STREM-1. sTREM-1 was detectable in the amniotic fluid of pregnant women with PROM while the serum STREM-1 level was very low (sTREM-1 was undetectable in the sera of 6 pregnant women). Moreover, amniotic fluid and serum concentrations of sTREM-1 were significantly higher in women with chorioamnionitis than concentrations in women without chorioamnionitis $(\mathrm{P}<0.001)$. Using Spearman rank correlation analysis, we showed that there was a marked correlation between serum and amniotic fluid sTREM-1 levels in pregnant women with PROM $\left(r_{s}=0.513, P<0.01\right)$. The results of serum and amniotic fluid concentrations of sTREM-1 in the two groups are presented in Table II and Fig. 1.

Serum CRP and WBC count. The levels of serum CRP and WBC count in pregnant women with PROM complicated with chorioamnionitis were significantly higher compared to the levels in the women without chorioamnionitis $(\mathrm{P}<0.05$, Table III). 
Table I. General information of the PROM pregnant women with or without concomitant chorioamnionitis.

\begin{tabular}{lcccr}
\hline Group & $\begin{array}{c}\text { Cases } \\
(\text { no. })\end{array}$ & $\begin{array}{c}\text { Age } \\
\text { (years })\end{array}$ & $\begin{array}{c}\text { Gestational age } \\
\text { (weeks) }\end{array}$ & $\begin{array}{c}\text { Birth weight } \\
(\mathrm{g})\end{array}$ \\
\hline Chorioamnionitis & 33 & $27(26-29)$ & $38.8(34.9-39.3)$ & $2980(2315-3422)$ \\
Non-chorioamnionitis & 22 & $27(25-27)$ & $38.1(35.9-39.2)$ & $2960(2556-3278)$ \\
P-value & & 0.292 & 0.973 & 0.631 \\
\hline
\end{tabular}

PROM, premature rupture of membranes. Values are represented as mean (range).

Table II. Comparison of serum and amniotic fluid concentrations of sTREM-1 in PROM pregnant women with or without chorioamnionitis.

\begin{tabular}{|c|c|c|c|}
\hline Group & $\begin{array}{l}\text { Cases } \\
\text { (no.) }\end{array}$ & $\begin{array}{l}\text { Serum sTREM-1 } \\
(\mathrm{pg} / \mathrm{ml})\end{array}$ & $\begin{array}{l}\text { Amniotic fluid sTREM-1 } \\
\qquad(\mathrm{pg} / \mathrm{ml})\end{array}$ \\
\hline Chorioamnionitis & 33 & $64.0(37.4-103.7)$ & $2053.8(1257.0-2750.5)$ \\
\hline Non-chorioamnionitis & 22 & $16.2(1.4-31.7)$ & 987.5 (861.6-1208.9) \\
\hline P-value & & 0.000 & 0.000 \\
\hline
\end{tabular}

sTREM-1, soluble triggering receptor expressed on myeloid cells-1; PROM, premature rupture of membranes. Values are represented as mean (range).

Table III. Levels of serum CRP and WBC count in the PROM pregnant women with or without chorioamnionitis.

\begin{tabular}{lccr}
\hline Group & $\begin{array}{c}\text { Cases } \\
(\text { no. })\end{array}$ & $\begin{array}{c}\text { CRP } \\
(\mathrm{mg} / \mathrm{l})\end{array}$ & $\begin{array}{c}\text { WBC } \\
\left(1 \times 10^{9} / 1\right)\end{array}$ \\
\hline Chorioamnionitis & 33 & $8.0(5.3-14.7)$ & $11.8(10.3-16.1)$ \\
Non-chorioamnionitis & 22 & $3.7(3.4-6.0)$ & $10.3(8.7-12.77)$ \\
P-value & & 0.001 & 0.018 \\
\hline
\end{tabular}

CRP, C-reactive protein; WBC, white blood cell; PROM, premature rupture of membranes. Values are represented as mean (range).

Comparisons of the diagnostic value of sTREM-1, WBC count and serum CRP for pregnant women with PROM complicated with subclinical chorioamnionitis. As shown by the analysis of the area under the ROC curve (AUC), serum sTREM-1 had an AUC of 0.785, a cutoff value of $31.4 \mathrm{pg}$ / $\mathrm{ml}$, a sensitivity of $81.8 \%$ and a specificity of $77.3 \%$ [95\% confidence interval (CI) 0.653-0.917, $\mathrm{P}<0.001$ ]; amniotic fluid sTREM-1 had an AUC of 0.865, a cutoff value of $1245 \mathrm{pg} /$ $\mathrm{ml}$, a sensitivity of $81.8 \%$ and a specificity of $86.4 \%(95 \%$ CI 0.764-0.966, $\mathrm{P}<0.001)$; WBC count had an AUC of 0.689 , a cutoff value of $10.5 \times 10^{9} / 1$, a sensitivity of $81.8 \%$ and a specificity of $54.5 \%(95 \%$ CI $0.544-0.835, \mathrm{P}=0.018)$; serum CRP had an AUC of 0.762, a cutoff value of $4.4 \mathrm{mg} / \mathrm{l}$, a sensitivity of $87.9 \%$ and a specificity of $68.2 \%(95 \% \mathrm{CI}$ 0.624-0.900, $\mathrm{P}=0.001)$. Comparisons of the AUCs of the 4 indicators demonstrated that amniotic fluid sTREM-1 and serum sTREM-1 achieved a statistical difference compared to the WBC count $(\mathrm{P}<0.05)$. Comparisons of the diagnostic value of sTREM-1, WBC count and serum CRP for PROM complicated with subclinical chorioamnionitis are shown in Fig. 2 and Table IV.

\section{Discussion}

The exact etiology of premature rupture of membranes remains elusive. Infection is suggested by many scholars as one of the main causes of PROM (9). Following rupture of the fetal membrane, the chance of ascending infection of the amniotic cavity by vaginal bacteria is significantly increased. Accordingly, infection and PROM are interdetermined. The present study revealed that the incidence of chorioamnionitis in combination with PROM was as high as $60 \%$ (33/55), indicating that chorioamnionitis correlates tightly with PROM. Additionally, none of the pregnant women with pathologically confirmed chorioamnionitis exhibited any signs of clinical infection, indicating that their infections were at the subclinical stage.

Subclinical chorioamnionitis poses a serious threat to the safety of mother and child by causing puerperal infection, 
Table IV. Comparison of the diagnostic value of sTREM-1, WBC and serum CRP for prediction of subclinical chorioamnionitis.

\begin{tabular}{lcccccc}
\hline Indicator & AUC & $\begin{array}{c}\text { Cutoff } \\
\text { value }\end{array}$ & $\begin{array}{c}\text { Sensitivity } \\
(\%)\end{array}$ & $\begin{array}{c}\text { Specificity } \\
(\%)\end{array}$ & $\begin{array}{c}\text { Positive predictive } \\
\text { value }(\%)\end{array}$ & $\begin{array}{c}\text { Negative predictive } \\
\text { value }(\%)\end{array}$ \\
\hline Serum sTREM-1 & $0.785^{\mathrm{a}}$ & $31.4(\mathrm{pg} / \mathrm{ml})$ & 81.8 & 77.3 & 84.4 & 73.9 \\
Amniotic sTREM-1 & $0.865^{\mathrm{a}}$ & $1245(\mathrm{pg} / \mathrm{ml})$ & 81.8 & 86.4 & 90.0 & 76.0 \\
CRP & 0.762 & $4.4(\mathrm{mg} / \mathrm{l})$ & 87.9 & 68.2 & 80.5 & 78.9 \\
WBC & 0.689 & $10.5 \times 10^{9} / 1$ & 81.8 & 54.5 & 70.6 & 57.1 \\
\hline
\end{tabular}

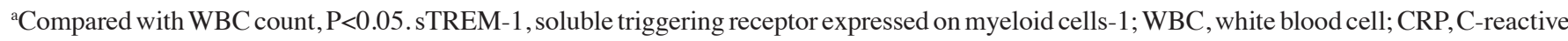
protein.

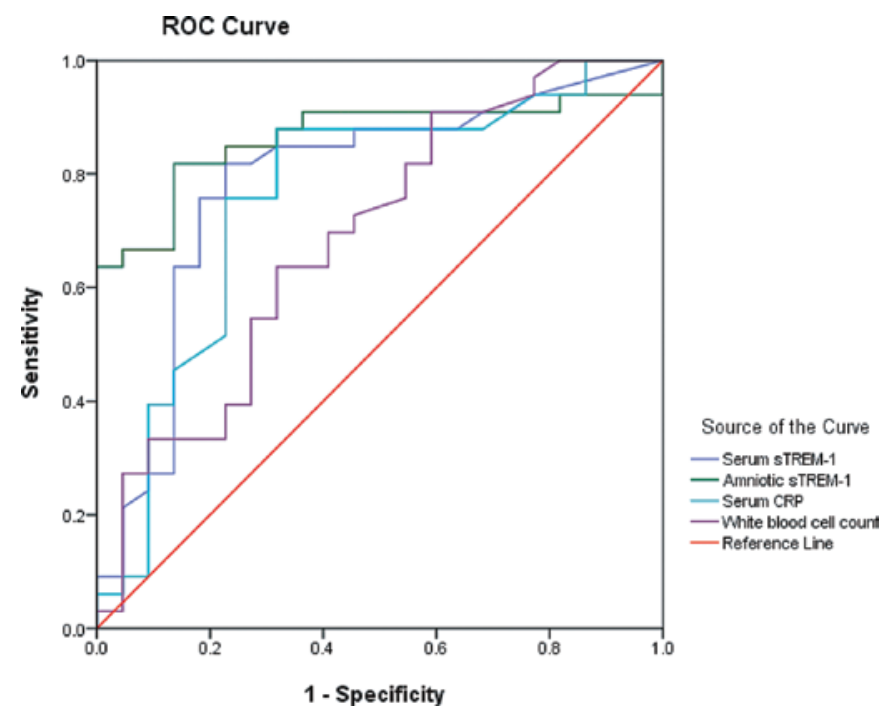

Figure 2. ROC curves of serum soluble triggering receptor expressed on myeloid cells-1 (sTREM-1), amniotic fluid STREM-1, white blood cell (WBC) count, and serum CRP for prediction of premature rupture of membranes (PROM) complicated with subclinical chorioamnionitis.

sepsis and other infectious diseases in infants (10). In order to identify sensitive and specific markers of infection, Ohlsson and Wang evaluated 23 relevant indicators and proposed that certain laboratory indicators such as WBC count, erythrocyte sedimentation rate and CRP have varying specificity and positive predictive value for the monitoring of intrauterine infection, but lack sufficient sensitivity and negative predictive value (11). Elevated WBC count is an effective indicator for serious systemic infection, but is non-specific. Previous studies suggest that WBC count is highly specific for severe infections, but offers limited value for monitoring histological chorioamnionitis. WBC count, particularly that of neutrophils, increases in the body during pregnancy. If hormones are used to promote maturation of fetal lungs, false elevation of WBCs may be observed. Therefore, WBC count cannot accurately reflect the development of infection and disease progression during pregnancy. CRP is an acute phase response protein synthesized by liver cells. It is capable of directly revealing tissue inflammation and necrosis, is a sensitive indicator of bacterial infection and could be used to monitor intrauterine infection (12). However, it is low in specificity. Numerous clinical factors may lead to false positivity of serum CRP, including liver disease, rheumatism, cancer and cardiovascular disease. Hence, it is necessary first to exclude these diseases when CRP is used for the diagnosis of chorioamnionitis. The present study also revealed that WBC count and serum CRP offered limited value for prediction and diagnosis of PROM complicated with subclinical chorioamnionitis. CRP has high sensitivity, but low specificity. WBC count also lacks sufficient specificity and sensitivity.

TREM-1 is a transmembrane glycoprotein that belongs to the immunoglobulin superfamily and is mainly expressed in neutrophils, monocytes and macrophages (13). It is generally believed that TREM-1 activates downstream signal transduction via the connection between its charged transmembrane domain and DAP12, thus activating ELK-1, NFAT, AP-1 and NF- $\kappa$ B and other transcription factors. These events finally lead to increased expression of TNF- $\alpha$, IL-1, IL-8, GM-CSF and other cytokines, while inhibiting the expression of the anti-inflammatory factor IL-10 (14). TREM-1 exists in two forms: cell surface TREM-1 and soluble sTREM-1. At present, the exact source of sTREM-1 remains controversial. Certain studies indicate that sTREM-1 is a soluble form of TREM-1 membrane protein shed into the body fluid from the surface of activated macrophages $(15,16)$. Others propose that sTREM-1 is a secretory isoform of a TREM-1 splice mutant that encodes no transmembrane domain (17). During infection, sTREM-1 is released into body fluid and is closely related to the severity of infection. A number of studies have revealed that STREM-1 is a significant marker for the diagnosis of inflammatory diseases and a novel early warning indicator of infectious diseases, demonstrating that it is more valuable for diagnosis and differential diagnosis than other clinical or laboratory markers (18-21). Menon et al and Kusanovic et al reported the presence of sTREM-1 in the amniotic fluid of term and preterm pregnant women, and that amniotic fluid concentrations of sTREM-1 were significantly higher in pregnant women with intra-amniotic infection than in those without, indicating that amniotic fluid sTREM-1 may play a role in intra-amniotic infection $(16,22)$.

In this study, sTREM-1 was detected in the amniotic fluid of all pregnant women with PROM, which is consistent with the above-mentioned findings (22). In addition, our study also found that amniotic fluid and serum concentrations of sTREM-1 were significantly higher in pregnant women with PROM complicated with subclinical chorioamnionitis compared to those without chorioamnionitis $(\mathrm{P}<0.001)$. This 
indicates that sTREM-1 may be involved in the immune response of intra-amniotic infection and play a crucial role in the pathogenesis and development of chorioamnionitis. We also noted that STREM-1 concentrations were significantly higher in the amniotic fluid than in serum, which may be due to the following causes. Firstly, TREM-1 activation and expression may be mainly localized in the placenta and fetal membranes. This means that following pathogen infection a large number of immune effector cells are recruited into the amniotic cavity, causing inflammation, whereas peripheral organs and blood may be less affected. Secondly, amnionitis lesions are mainly confined to the amniotic cavity, and TREM-1 expression also mainly occurs in the amniotic cavity. Due to the placental barrier, sTREM-1 is excreted with difficulty, and accumulates in the amniotic cavity, rendering sTREM-1 concentrations in the amniotic cavity significantly higher than in the serum.

Early diagnosis of PROM complicated with subclinical chorioamnionitis is difficult in clinical practice. This study found that serum and amniotic fluid STREM-1 levels were significantly higher in pregnant women with PROM complicated with chorioamnionitis compared to those without, and that WBC count and serum levels of CRP were also significantly higher compared to those without chorioamnionitis. ROC analysis revealed that the AUC using serum and amniotic fluid sTREM-1 concentrations for prediction of subclinical chorioamnionitis was 0.865 and 0.785 , respectively, significantly greater compared to that with WBC count, indicating that serum and amniotic fluid sTREM-1 concentrations are superior to $\mathrm{WBC}$ count in predictive value. The most appropriate cutoff values of various indicators obtained with the ROC curve indicate that serum and amniotic fluid sTREM-1 concentrations have similar sensitivity in predicting subclinical chorioamnionitis with WBC count and serum CRP. However, when examined in terms of specificity, amniotic fluid sTREM-1 $>$ serum STREM-1 > serum CRP > WBC count, and serum and amniotic fluid sTREM-1 offer higher positive and negative predictive value than WBC count and serum CRP. Therefore, serum and amniotic fluid sTREM-1 levels may emerge as new indicators for prediction of PROM complicated with chorioamnionitis, and may prove to be particularly beneficial for early prediction of subclinical infection of pregnant women.

Clinically, it is relatively complex to collect amniotic fluid samples whereas blood sample collection is simple, convenient and repeatable. Therefore, monitoring of serum sTREM-1 levels is more practical in clinical practice. Further elucidation of the action mechanism of sTREM-1 in inflammation and the relationship between chorioamnionitis and serum and amniotic fluid sTREM-1 levels is warranted.

\section{Acknowledgements}

This study was supported by grants from the Health Department of Zhejiang Province (2009A147) and the Science Foundation of Zhejiang Province of China (Y2110522).

\section{References}

1. Liu J, Feng ZC and $\mathrm{Wu} \mathrm{J}$ : The incidence rate of premature rupture of membranes and its influence on fetal-neonatal health: a report from mainland China. J Trop Pediatr 56: 36-42, 2010.
2. Gomez R, Romero R, Edwin SS and David C: Pathogenesis of preterm labor and preterm premature rupture of membranes associated with intraamniotic infection. Infect Dis Clin North Am 11: 135-176, 1997

3. Yoon BH, Romero R, Park JS, Kim CJ, Kim SH, Choi JH and Han TR: Fetal exposure to an intra-amniotic inflammation and the development of cerebral palsy at the age of three years. Am J Obstet Gynecol 182: 675-681, 2000.

4. Hannah ME, Ohlsson A, Farine D, Hewson SA, Hodnett ED, Myhr TL, Wang EE, Weston JA and Willan AR: Induction of labor compared with expectant management for prelabor rupture of the membranes at term. TERMPROM Study Group. N Engl J Med 334: 1005-1010, 1996.

5. Novak-Antolic Z, Pajntar M and Verdenik I: Rupture of the membranes and postpartum infection. Eur J Obstet Gynecol Reprod Biol 71: 141-146, 1997.

6. Romero R, Yoon BH, Mazor M, Gomez R, Gonzalez R and Diamond MP: A comparative study of the diagnostic performance of amniotic fluid glucose, white blood cell count, interleukin-6, and gram stain in the detection of microbial invasion in patients with preterm premature rupture of membranes. Am J Obstet Gynecol 169: 839-851, 1993.

7. ACOG Committee on Practice Bulletins-Obstetrics: ACOG Practice Bulletin No. 80: premature rupture of membranes. Clinical management guidelines for obstetrician-gynecologists. Obstet Gynecol 109: 1007-1019, 2007.

8. Nathan C and Ding A: TREM-1: a new regulator of innate immunity in sepsis syndrome. Nat Med 7: 530-532, 2001.

9. Romanik M and Martirosian G: [Frequency, diagnostic criteria and consequences of bacterial vaginosis in pregnant women]. Przegl Epidemiol 58: 547-553, 2004 (In Polish).

10. Bashiri A, Burstein E and Mazor M: Cerebral palsy and fetal inflammatory response syndrome: a review. J Perinat Med 34: $5-12,2006$.

11. Ohlsson A and Wang E: An analysis of antenatal tests to detect infection in preterm premature rupture of the membranes. Am J Obstet Gynecol 162: 809-818, 1990.

12. Van der Ham DP, van de Laar R, Mol BW and Willekes C: Use of C-reactive protein as a predictor of chorioamnionitis in preterm prelabour rupture of the membranes: a systematic review. BJOG 115: 127, 2008.

13 Chen LC, Laskin JD, Gordon MK and Laskin DL: Regulation of TREM expression in hepatic macrophages and endothelial cells during acute endotoxemia. Exp Mol Pathol 84: 145-155, 2008.

14. Bouchon A, Facchetti F, Weigand MA and Colonna M: TREM-1 amplifies inflammation and is a crucial mediator of septic shock. Nature 410: 1103-1107, 2001.

15. Mahdy AM, Lowes DA, Galley HF, Bruce JE and Webster NR: Production of soluble triggering receptor expressed on myeloid cells by lipopolysaccharide-stimulated human neutrophils involves de novo protein synthesis. Clin Vaccin Immunol 13: 492-495, 2006.

16. Menon R and Fortunato SJ: Induction of triggering receptors of myeloid cell (TREM-1) expression in fetal membranes and higher concentration of soluble TREM-1 in amniotic fluid with spontaneous preterm birth. Reprod Sci 15: 825-830, 2008.

17. Gomez-Pina V, Soares-Schanoski A, Rodriguez-Rojas A, Del Fresno C, Garcia F, Vallejo-Cremades MT, FernandezRuiz I, Arnalich F, Fuentes-Prior P and Lopez-Collazo E: Metalloproteinases shed TREM-1 ectodomain from lipopolysaccharide-stimulated human monocytes. J Immunol 179: 4065-4073, 2007.

18. Cohen J: TREM-1 in sepsis. Lancet 358: 776-778, 2001.

19. Bleharski JR, Kiessler V, Buonsanti C, Sieling PA, Stenger S, Colonna $\mathrm{M}$ and Modlin RL: A role for triggering receptor expressed on myeloid cells-1 in host defense during the early-induced and adaptive phases of the immune response. J Immunol 170: 3812-3818, 2003.

20. Gibot S: Soluble triggering receptor expressed on myeloid cells and the diagnosis of pneumonia and severe sepsis. Semin Respir Crit Care Med 27: 29-33, 2006.

21. Vogel I, Goepfert AR, Thorsen P, Skogstrand K, Hougaard DM, Curry AH, Cliver S and Andrews WW: Early second-trimester inflammatory markers and short cervical length and the risk of recurrent preterm birth. J Reprod Immunol 75: 133-140, 2007.

22. Kusanovic JP, Romero R, Chaiworapongsa T, et al: Amniotic fluid sTREM-1 in normal pregnancy, spontaneous parturition at term and preterm, and intra-amniotic infection/inflammation. J Matern Fetal Neonatal Med 23: 34-47, 2010. 